\title{
Solids transport of non-Newtonian slurries in Iaminar open channel flow
}

\author{
R.H. Hernández LEAF-NL, Departamento de Ingeniería Mecánica, Universidad de Chile, Chile
}

R.H. Fuentes J.R.I. Ingeniería, Chile

\section{Abstract}

Numerically the authors solved the Navier Stokes equations in two dimensions coupled with a highly nonlinear advection-diffusion equation which predicts the volume fraction of solids in highly concentrated mono-modal suspensions. Our main purpose is to simulate the solid transport of non-Newtonian slurries of high concentration in laminar 2D open channel and duct flows.

The results of the $2 D$ numerical simulation confirm that particle migration effectively occurs from high to low shear regions, provided that the sedimentation flux is smaller compared to other particle migration mechanisms (Phillips et al., 1992).

\section{Introduction}

By reasons of water recovery and huge throughput of modern mining operations, there is an increasing need for solids transport in high concentrations. Often high concentration implies non-Newtonian flow of slurries, where segregation may be developed. The aim of the present work is to predict the flow behaviour observed in the transport of slurry flows like the thickened tailings produced by the mining industry, especially in the case of non-Newtonian laminar flow. These slurries typically exhibit a non-Newtonian rheological behaviour, in part determined by the particle size distribution and local shear. To address such non-linear dynamics, the authors have developed a 2D numerical model to predict the flow field of high concentration slurries of coarse solid particles with non-Newtonian rheological properties in open channels and ducts.

The results show that the model predicts a realistic 2D laminar flow field inside the open channel and it is possible to compute the particle concentration in the overall domain, showing the downstream advection and diffusion of particle spots of high concentration, combined with particle settling, depending on particle size and relative density as well as yield stress. Good agreement was found between the results with the numerical simulations and experiments of Spelay (2007), and the theoretical results of Phillips et al. (1992) and Mills and Snabre (1995).

\section{Methodology}

The authors solved the generalised version of the Navier Stokes and mass conservation equations coupled with a scalar concentration equation to describe the average behaviour of coarse mono-modal particles in laminar flows. The scalar concentration equation predicts the volume fraction of solid particles, and allows computation of the apparent viscosity of the fluid as a function of both local solid concentration and local shear, which is finally considered as the input to the stress tensor of the Navier Stokes equations.

The starting point of the model is the shear-induced particle migration theory of Phillips et al. (1992); a constitutive advection-diffusion equation to determine particle concentration as a result of shear-induced effects. The final apparent viscosity model is an explicit function of particle concentration and local shear rate, which can be written as a bi-viscosity Bingham fluid model by Beverly and Tanner (1992). Alternatively, when single dependence on particle concentration was needed, the authors considered the models of Krieger (1972) and Mills and Snabre (1995). 
The governing momentum and concentration partial differential equations were solved numerically in primitive variables. In the discretisation of the open channel domain, the authors used a 2D, uniform and staggered grid under a control volume formulation, and incorporated the SIMPLER algorithm of Patankar (1980) with a power law scheme already tested by Hernández (1995) to manage advection-diffusion terms in 2D and 3D discrete models. The discrete equations were solved by an iterative tri-diagonal solver (TDMA) with additional criteria for fast convergence and no-slip conditions at the solid boundaries. Special consideration was taken of the conditions fulfilled by the boundary conditions for the concentration equation to avoid particle flux at the solid and free boundaries.

\section{1 Governing equations}

Mass conservation equation is:

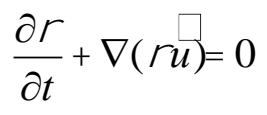

Momentum conservation equation is:

$$
\left(\frac{\partial u}{\partial t}+u \cdot \nabla u\right)=\nabla p+\stackrel{\square}{g}+\nabla \cdot\left(2 d \frac{2}{3}(\nabla \cdot u) I\right)
$$

where the $d$ is the tensor given by:

$$
d_{i j}=\frac{1}{2} \frac{u_{i}}{x_{j}}+\frac{u_{j}}{x_{i}} \underset{\doteqdot}{\doteqdot}
$$

The 2D computational domain is represented in Figure 1 as a channel or duct of length $L$ and height $d$.

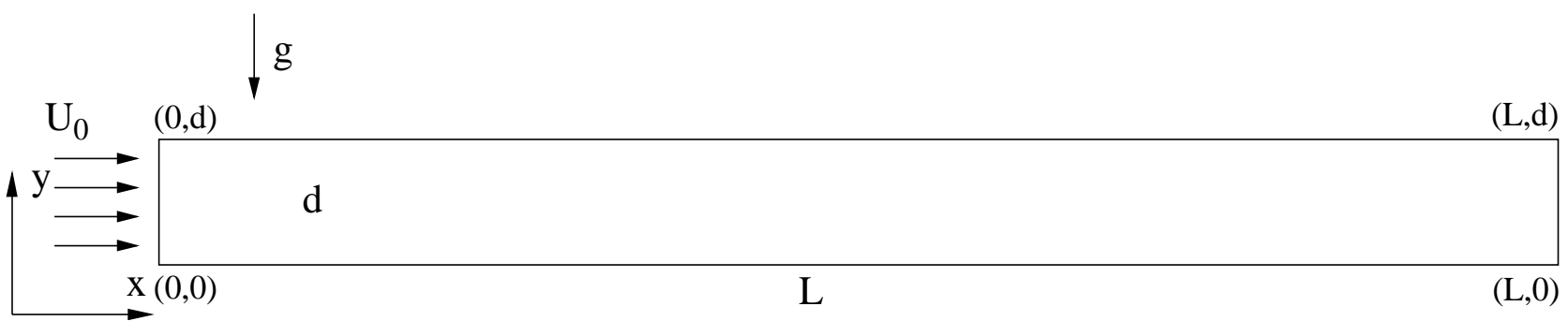

Figure 1 Schematics of computational domain of height $d$, length $L$ and slope $2 \%$. Inlet flow velocity is $U_{0}$ at $x=0$. The carrier fluid is a Bingham fluid of plastic viscosity $\mu_{p}$, with yield stress $\tau_{0}$ and density $\rho$

\section{2 Scalar transport equation}

The apparent viscosity is a function of shear rate $[$ and volume fraction . To compute the volume fraction the authors introduce the Phillips model (Phillips et al., 1992) which solves the volume fraction of solid particles as a diffusion-advection equation with three fluxes as the forcing mechanism for particle migration.

The conservation equation written in primitive variables reads:

$$
\frac{\partial}{\partial t}+u \cdot \nabla=\nabla \cdot\left(N_{c}+N+N_{g}\right)
$$

where the fluxes (Phillips et al., 1992; Spelay, 2007) are given in Table 1. 
Table 1 Particle migration mechanisms

\begin{tabular}{ll} 
Flux due to particle sedimentation & $N_{g}=K_{g} a^{2} f() \bar{g}$ \\
Flux due to varying collision frequency & $N_{c}=K_{c} a^{2} \nabla(\square)$ \\
Flux due to spatially varying viscosity & $N=K a^{2} \sqsubset^{2} \underline{\nabla}$ \\
\hline
\end{tabular}

The two parameters $K_{\mathrm{c}}$ and $K_{\mathrm{n}}$ are proportionality constants (Spelay, 2007) determined in the work of Phillips et al. (1992) from a comparison with their experimental results. A ratio of $K_{c} / K_{n}=0.66$ provides good results (it never should exceed 1). The rest of the parameters are the particle size $a$, the gravity parameter $K_{\mathrm{g}}$ which is defined by (Spelay, 2007):

$$
K_{g}=\frac{2}{9}\left(\begin{array}{ll}
s & f
\end{array}\right)
$$

and the hindrance function $f(\quad)$ which is defined by:

$$
f()=\underline{(1 \quad)}
$$

\section{3 Bingham fluid model}

The non-Newtonian slurry is modelled as a Bingham fluid where the shear stress is given by:

$$
={ }_{0}+{ }_{p}[
$$

The apparent viscosity is then defined by the following relationship:

$$
=\frac{0}{\sqsubset}+p
$$

where the shear rate is computed as the second invariant of the deformation tensor ${ }_{i j}$ :

$$
\tau=\left(\frac{1}{2} \quad:\right)^{1 / 2}
$$

To avoid divergences of the apparent viscosity at very small shear rates, the authors use the bi-viscosity model (Beverly and Tanner, 1992) which assumes that the rheology of the fluid at lower shear rates is Newtonian but with a viscosity two or three orders of magnitude greater than the Bingham plastic viscosity.

$$
=\begin{array}{cc}
\frac{0}{\square}+{ }_{p} & \square \varpi_{\bar{c}} \\
0 & \text { otherwise }
\end{array}
$$

where each term is defined as follows:

$$
\bar{c}_{\bar{c}}=\frac{0}{\left(\begin{array}{ll}
0 & p
\end{array}\right)} \text { with } \quad{ }_{0}=10^{3}
$$


This dual behaviour can be understood in Figure 2. There is a critical shear rate, $\square_{c}$ separating two regions of different slope in the linear relationship between shear stress and shear rate.

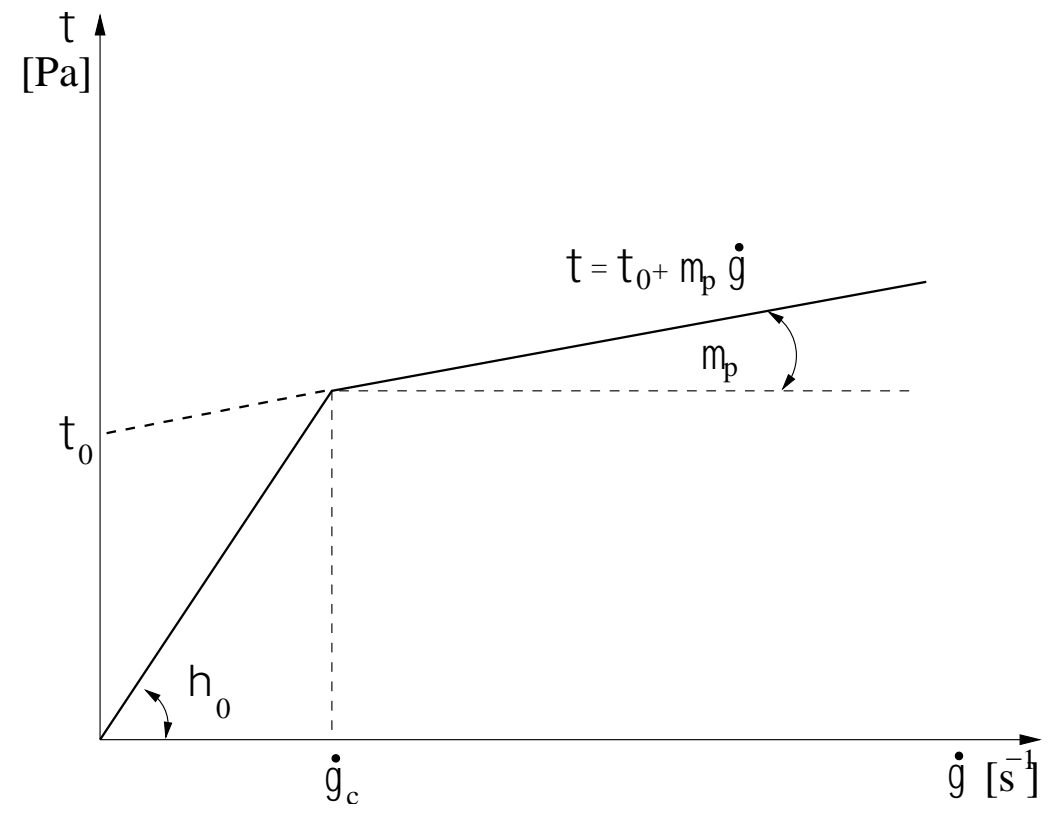

Figure 2 Bi-viscosity model

The apparent viscosity is then bounded by an upper limit when the flow shear thinning mechanism is unable to overcome the yield stress of the fluid.

In Figure 3 the authors plotted the typical bi-viscosity behaviour as will normally occur during the numerical computation inside the fluid domain.

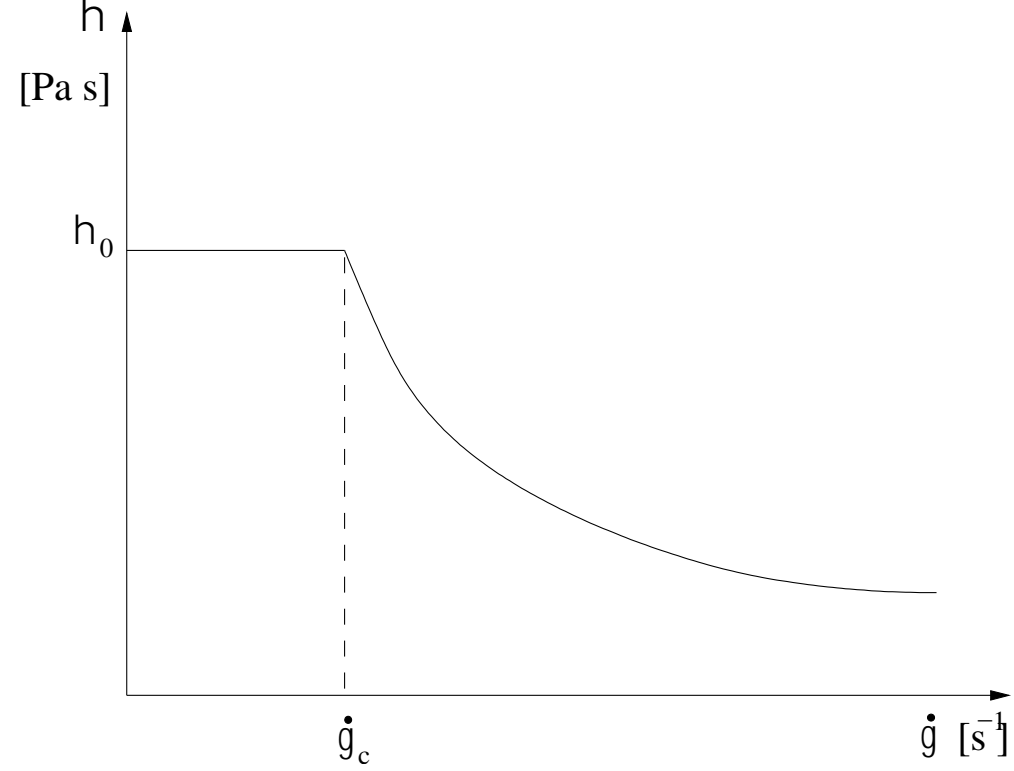

Figure 3 The apparent viscosity as a function of the shear rate calculated with the bi-viscosity mode I

Below the critical shear rate $\zeta_{c}$, the apparent viscosity becomes very high, $={ }_{0}$. Since this model avoids the divergent behaviour of apparent viscosity at low shear rates in a very simple way, it has been incorporated in the numerical simulation (Spelay, 2007). 
There exist many other apparent viscosity models in the literature, like the Carreau or Papanastasiou models, but the computational cost is too high to be considered here.

\section{4 Volume fraction and viscosity}

In our formulation for concentrated systems we can incorporate three different models for the dependence between the relative viscosity ${ }_{r}$ with the volume fraction .

The first model choice is the empirical Krieger's model (Krieger, 1972) for Newtonian systems, where the relative viscosity dependence with solids concentration is given by:

$$
r()=1-\div_{m}^{1.82}
$$

where $\quad m$ is the maximum packing concentration allowed. The Phillips work on concentrated systems (Phillips et al., 1992) is based on this equation.

The second model is from the work of Mills and Snabre (1995), described by the following equation:

$$
r()=\frac{(1)}{\left(1 /{ }_{m}\right)^{2}}
$$

which can be used with Newtonian systems.

The third and more complete model is the one formulated by Schaan et al. (2000) using the linear concentration ( )

$$
r()=1+2.5+0.16^{2} \text { where }=\frac{m}{\frac{1}{3}} \div^{1} 1^{1}
$$

Within the framework of this third model, the final apparent viscosity can be written as a function of shear rate and volume fraction for both Newtonian and non-Newtonian systems:

$$
\left(\ulcorner)=\min \frac{0}{\sqsubset},_{0} \div+{ }_{p} r()\right.
$$

If the volume fraction is zero, then the relative viscosity is unity and the authors came back to a pure biviscosity Bingham model.

\section{5 Boundary conditions}

To solve this highly non-linear hydrodynamic problem, a careful selection of the boundary conditions should be accomplished. In the case of flow velocity, to solve the Navier Stokes equations, the no-shear and no-slip conditions at the free surface and walls respectively, can be easily implemented.

However, within the Phillips equation, strict care must be taken when solving the volume fraction. We can use Dirichlet and a no-flux condition at free and solid walls. The non-flux condition prevents solid particles escaping from the domain through the walls during the transient of the simulation.

The no-flux condition can be expressed as:

$$
\left(N_{c}+N+N_{g}\right) \times \hat{n}=0
$$


which in turn can be written in explicit form in terms of the spatial derivative of the volume fraction:

$$
\frac{d}{d y}=\frac{K_{g} f() g_{y} \quad K_{c} \frac{d \sqsubset}{d y}}{\sqsubset K_{c}+K-\frac{d}{d} \div}
$$

\section{$3 \quad$ Numerical model}

The authors have developed a 2D numerical model to simulate the hydraulic transport of solid particles in a non-Newtonian laminar fluid in open channels and ducts as an extension of the work of Spelay (2007).

The governing equations were solved in primitive variables. The physical domain (channel or duct depending on the choice of the top boundary condition) was represented with a Cartesian 2D, uniform and staggered grid within a control volume formulation (finite volume), structured under the revised version of the SIMPLE algorithm of Patankar (1980). A power law scheme was used (Hernández, 1995) to manage the convective-diffusive terms in the discrete formulation of both; the Navier Stokes equation and the advection diffusion equation for the volume fraction.

The discrete equations were solved by an iterative TDMA with additional criteria for fast convergence (Van Doormaal and Raithby, 1984). The time step $\Delta \mathrm{t}$ used in all calculations was $t=10^{3} \mathrm{~s}$. A steady state solution required around $10^{4}$ time steps; an equivalent of approximately one hour of CPU on a 4-core based on Xeon processors. To ensure convergence of the numerical algorithm at each time step, the following convergence criteria was applied to all dependent variables at any grid location:

$$
\left|\begin{array}{cc}
\begin{array}{cc}
m \\
i j
\end{array} & \begin{array}{c}
m+20 \\
i j
\end{array} \\
\hline & m \\
& i j
\end{array}\right| 10^{5}
$$

Where represents a dependent variable, the indexes $(i, j)$ indicate a grid point, and the index $(m)$ a given iteration of the computer code. The maximum residue of the discrete volume fraction equation and momentum equations satisfied this convergence criteria (Hernández, 1995). In addition, the authors performed an internal global check of accuracy and consistency with an overall balance of momentum fluxes across the domain. This shows an overall residue of always below $2 \%$ of the incoming momentum flux at the bottom wall when steady state solutions arise.

However, in some cases this residue was found to be less than $1 \%$. If the convergence criteria is stretched, this residue can be minimised (up to machine precision), but the number of iterations required to solve the equations at a given time step increases notably.

In this work uniforms grids of $20 \times 2,000$ spatial points were used with $\Delta x=\Delta y=5 \mathrm{~mm}$. A finer grid of $40 \times 4,000$ points was also tested with a smaller step $\Delta x=\Delta y=2.5 \mathrm{~mm}$ to check if varying the grid spacing could increase the accuracy of the calculations. The results indicate a relative difference from the first grid of $2.5 \%$ in the averaged skin friction co-efficient calculated as:

$$
C_{f}=\frac{w}{\frac{1}{2} U_{0}^{2} \div}
$$

along the bottom wall, which demonstrates an increased accuracy with finer grids. However, differences in velocity profiles were under $3 \%$. The required computational time to reach the steady state solution with the finest grid suggests that the choice of the first grid to perform the calculations represents a good compromise between accuracy and computational effort. 


\section{$4 \quad$ Results}

\section{1 Duct flow of non-buoyant particles}

The authors performed the first simulation in a 2D duct with neutrally buoyant particles of size a = 300 $m$, in order to have an approximated closer look of the laminar flow and the further effect of the Phillips fluxes $N_{c}$ and $N$ at high average volume fractions \langle\rangle . The authors do not consider the effect of gravity $(g=0)$ in the present section. The simulation starts with a steady state flow and an inlet average concentration $(0, y)={ }_{0}=\langle\rangle$.

In Figure 4 the authors display the steady state velocity profiles $V(x=L, y)$ developed downstream, a duct of height $d=0.1 \mathrm{~m}$ and length $\mathrm{L}=10 \mathrm{~m}$ with neutrally buoyant particles of size $300 \mathrm{~m}$.

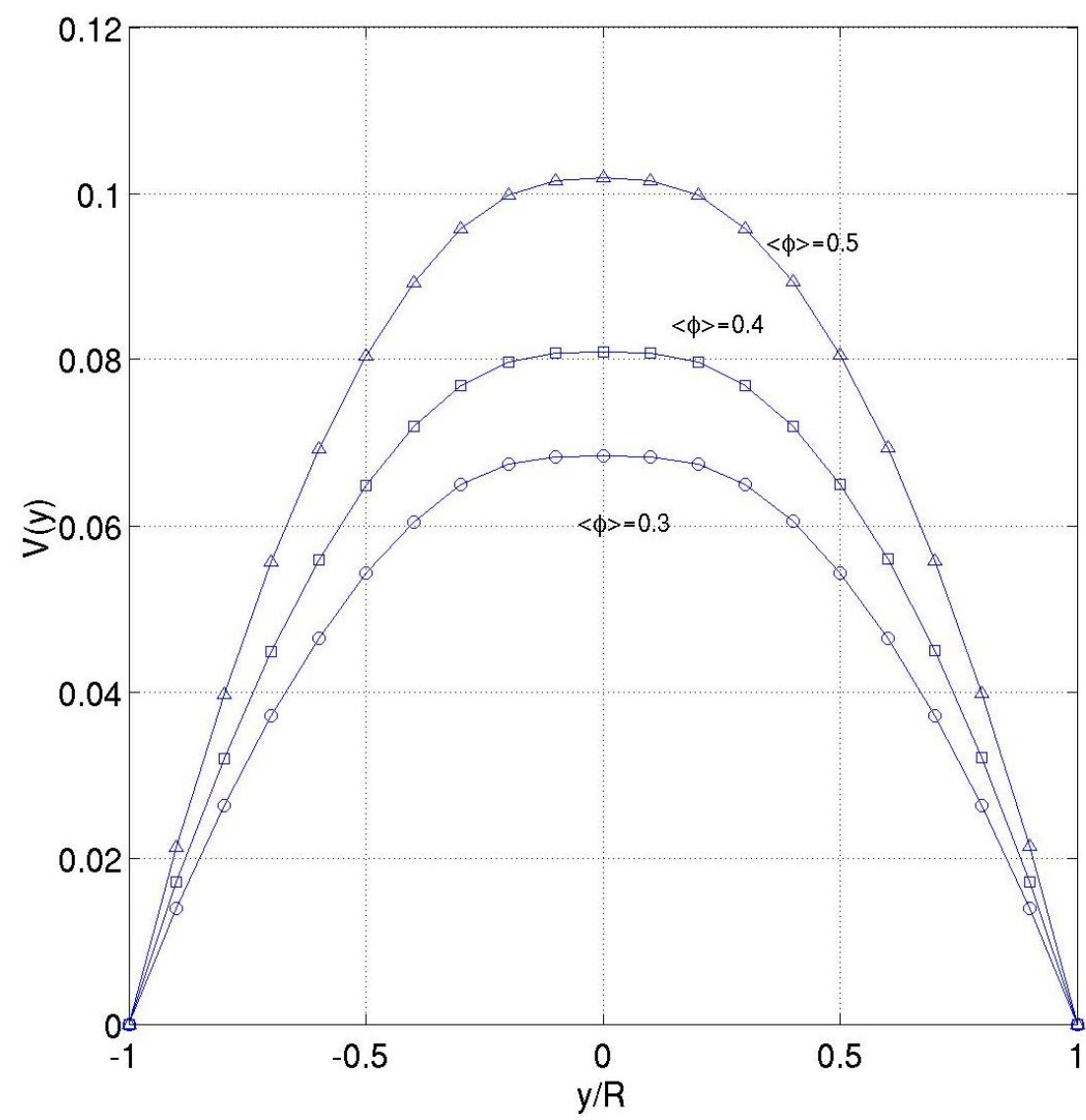

Figure 4 Velocity profiles at the duct exit $(d=2 R)$ for neutrally buoyant particles of size a $=300$ $m$. Flow velocity is $\mathrm{U}_{0}=0.1 \mathrm{~m} / \mathrm{s}$, Bingham viscosity $p=0.268$ Pas, yield stress is ${ }_{0}=2.67 \mathrm{~Pa}$, packing maximum is ${ }_{m}=0.68$, and density is $=2,790 \mathrm{~kg} / \mathrm{m}^{3}$

The velocity and volume fraction profiles are similar to the results of Phillips (Phillips et al., 1992) with similar concentrations. However, in our case, as the authors solve a 2D model, we get also the downstream motion of the particles and flow, and therefore the particle concentration profiles in the downstream direction.

The authors can follow the progression of the scalar concentration downstream (and in time) as it is shown in Figure 5. The authors observe an increase of concentration at the centreline of the duct that is accompanied with a decrease of concentration near the wall (Figure 6.) 
This is explained by the fact that there is a flux of particles away from the duct walls, towards the centreline of the flow, promoted by the Phillips fluxes. This local increase of produces an increase of apparent viscosity of the mixture which in turn produces the blunt-nose velocity profiles of Figure 4.

Another important point is the downstream progression of the particle concentration. In Figure 5 the authors show the 2D colour map of particle concentration in terms of volume fraction $(x, y)$ on the 2D duct flow after the steady state is achieved. The concentration of neutrally buoyant particles of size $300 m$ increases in the centre of the duct and starts earlier in the downstream coordinate when the starting average concentration is higher.
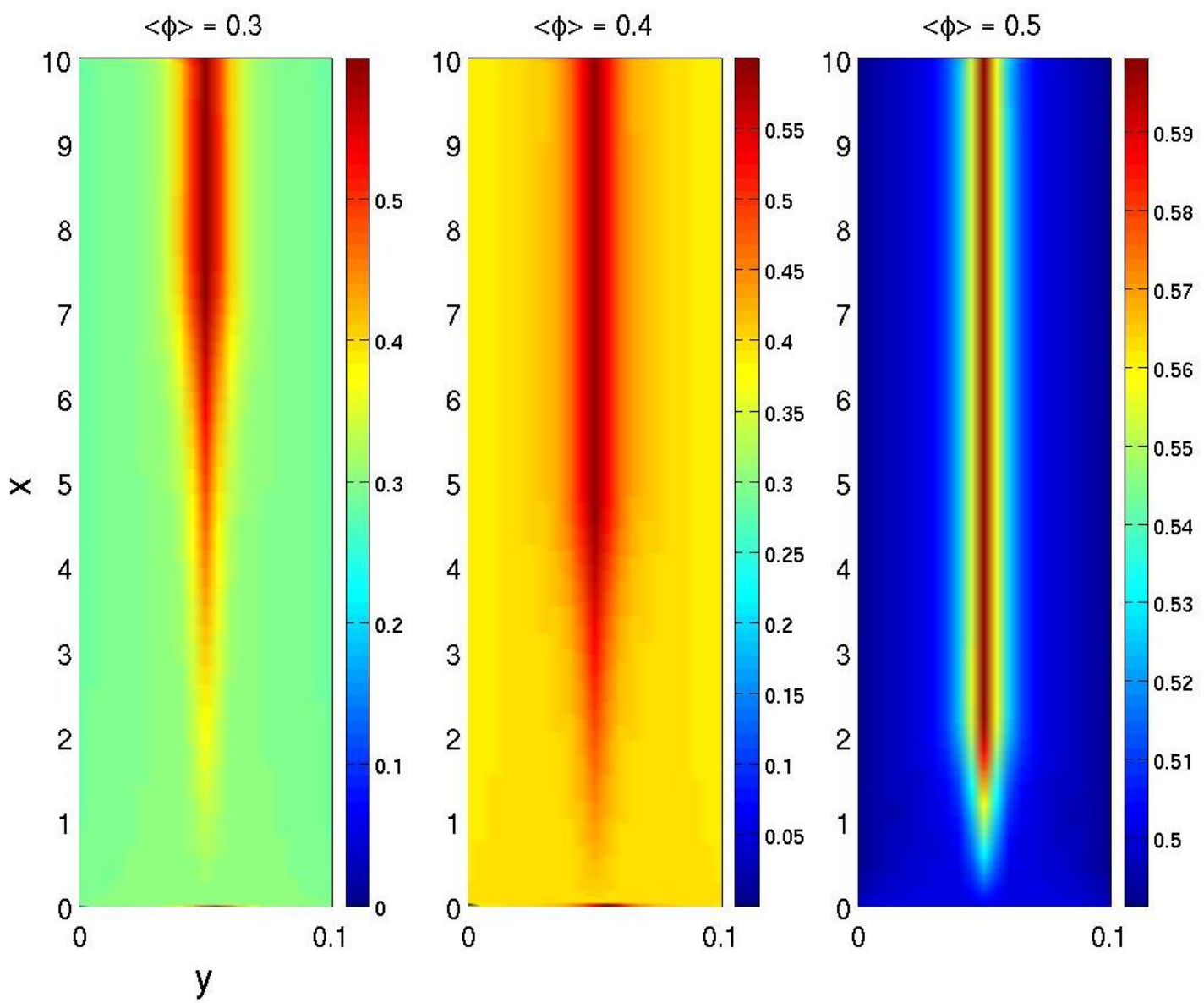

Figure 5 Concentration contours for neutrally buoyant particles of size a $=300 \mathrm{~m}$ (not to scale). Duct in $\mathrm{m}$, inlet flow velocity is $\mathrm{U}_{0}=0.1 \mathrm{~m} / \mathrm{s}$, Bingham viscosity ${ }_{p}=0.268$ Pas, yield stress is ${ }_{0}=2.67 \mathrm{~Pa}$, packing maximum is ${ }_{m}=0.68$ and density is $=2,790 \mathrm{~kg} / \mathrm{m}^{3}$

The increase of volume fraction at the duct axis obeys the migration of coarse particles due to the flux $N$, by spatial gradients of apparent viscosity, and the flux $N_{c}$ by spatial gradients of shear rate and concentration.

A clearer picture of the particle migration is shown in Figure 6 where the authors display the steady state concentration profiles $(x=L, y)$ developed downstream the duct of height $\mathrm{d}=0.1 \mathrm{~m}$ and length $\mathrm{L}=10 \mathrm{~m}$ with neutrally buoyant particles of size $\mathrm{a}=300 \mathrm{~m}$.

The authors determine the increase of volume fraction at the duct axis where the shear rate is zero. Without gravity, coarse particle migration into the duct axis is determined by the Phillips fluxes balance only, increasing the volume fraction up to maximum packing. Observe that the volume fraction decreases near the wall where the shear rate is maximum. 


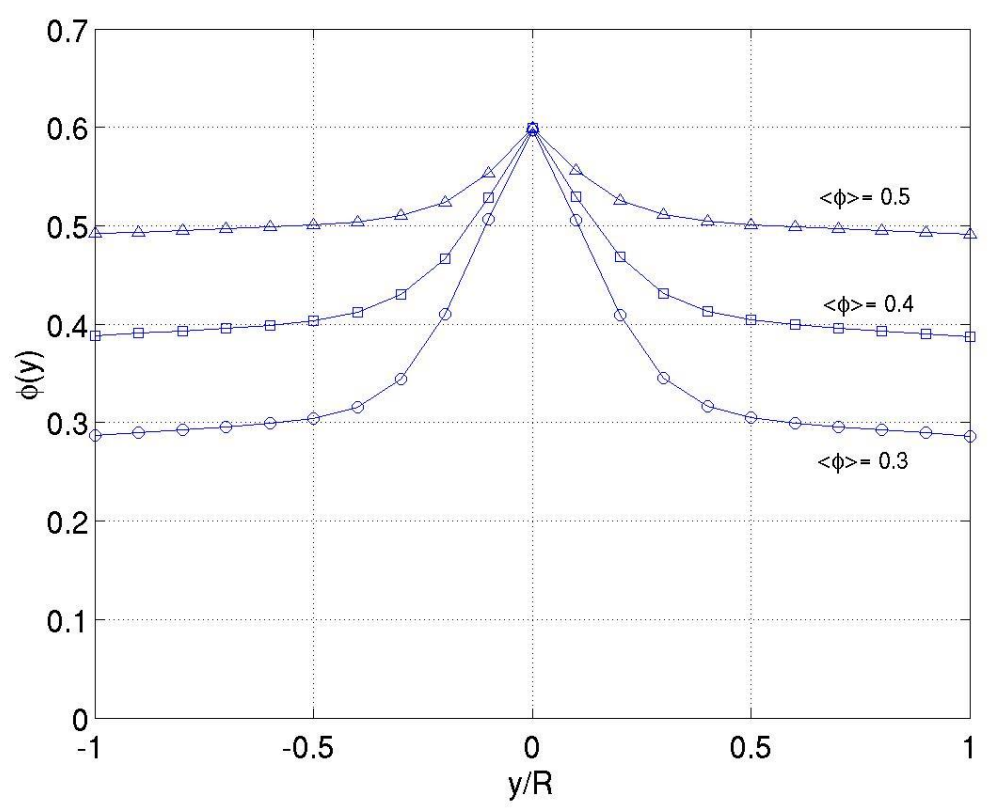

Figure 6 Concentration profiles at the duct exit for neutrally buoyant particles of size $a=300$ $m$. Inlet flow velocity is $U_{0}=0.1 \mathrm{~m} / \mathrm{s}$, Bingham viscosity $p=0.268$ Pas, yield stress is ${ }_{0}=2.67 \mathrm{~Pa}$, packing maximum is ${ }_{m}=0.68$ and density is ${ }^{p}=2,790 \mathrm{~kg} / \mathrm{m}^{3}$

\section{2 Open channel flow prediction}

A number of numerical simulations were performed in the case of channel flow with gravity, $g_{y}=-9.8 \mathrm{~m} / \mathrm{s}^{2}$. Using a channel height of $d=0.5 \mathrm{~m}$ and length of $\mathrm{L}=10 \mathrm{~m}$, the authors modelled the flow field of a Bingham slurry fluid of high volume concentration (thickened tailings).

Here, the numerical simulation also starts with a steady state flow and an inlet average concentration $(0, y)={ }_{0}$.

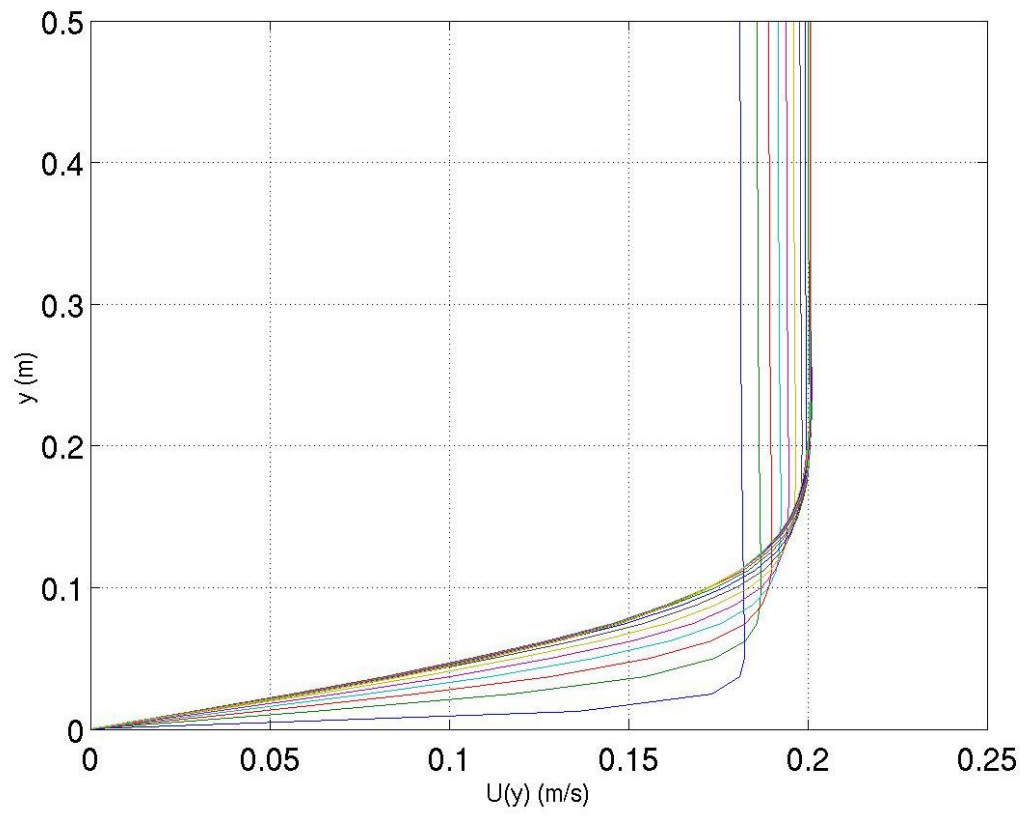

Figure 7 Typical evolution of channel flow velocity profiles along the downstream coordinate $x$. The channel inlet flow velocity at $\mathrm{x}=0$ is uniform $U_{0}=0.17 \mathrm{~m} / \mathrm{s}$. Bingham viscosity is ${ }_{p}=$ 0.268 Pas, ${ }_{0}=2.67 \mathrm{~Pa}, \quad{ }_{m}=0.68$ and $=2,790 \mathrm{~kg} / \mathrm{m}^{3}$ 
Figure 7 shows the steady state velocity profiles at different downstream positions ( $x=1 \mathrm{~m})$ showing the boundary layer shape but also the downstream self-evolution. An unsheared plug (upper layer $y \quad 0.1$ ) above the sheared layer ( $\left.\begin{array}{lll}y & 0.1 & \mathrm{~m}\end{array}\right)$ develops as expected. According to the theory, the behaviour of a spot of particles feed into these two layers will be different as the Phillips fluxes will be turned on and off in the lower and upper layers respectively.

The next two figures show the behaviour of the diffusion equation solved in the channel flow with a localised spot of initial volume fraction $(x, y)=0.5$. It seems to be of great interest that the equation can model the effect of the three main fluxes involved in the Phillips equation very well, in particular the action of the sedimentation flux observed in time on the local particle spot, when the channel flow is already well developed.

The numerical simulation starts with a steady state Bingham fluid channel flow of same height $d=0.5 \mathrm{~m}$, but the authors need to pay attention to the sheared layer only $\left(\begin{array}{lll}y & 0.1 \mathrm{~m}\end{array}\right)$. The authors define a small centred rectangular region $(0.040 .04 \mathrm{~m})$ where a particle spot is put inside the sheared layer of the channel velocity profile, near the inlet with a desired volume fraction, $(x, y)=0.5$ (red), which evolves by the effect of combined mechanisms of diffusion, sedimentation and advection by the main flow.

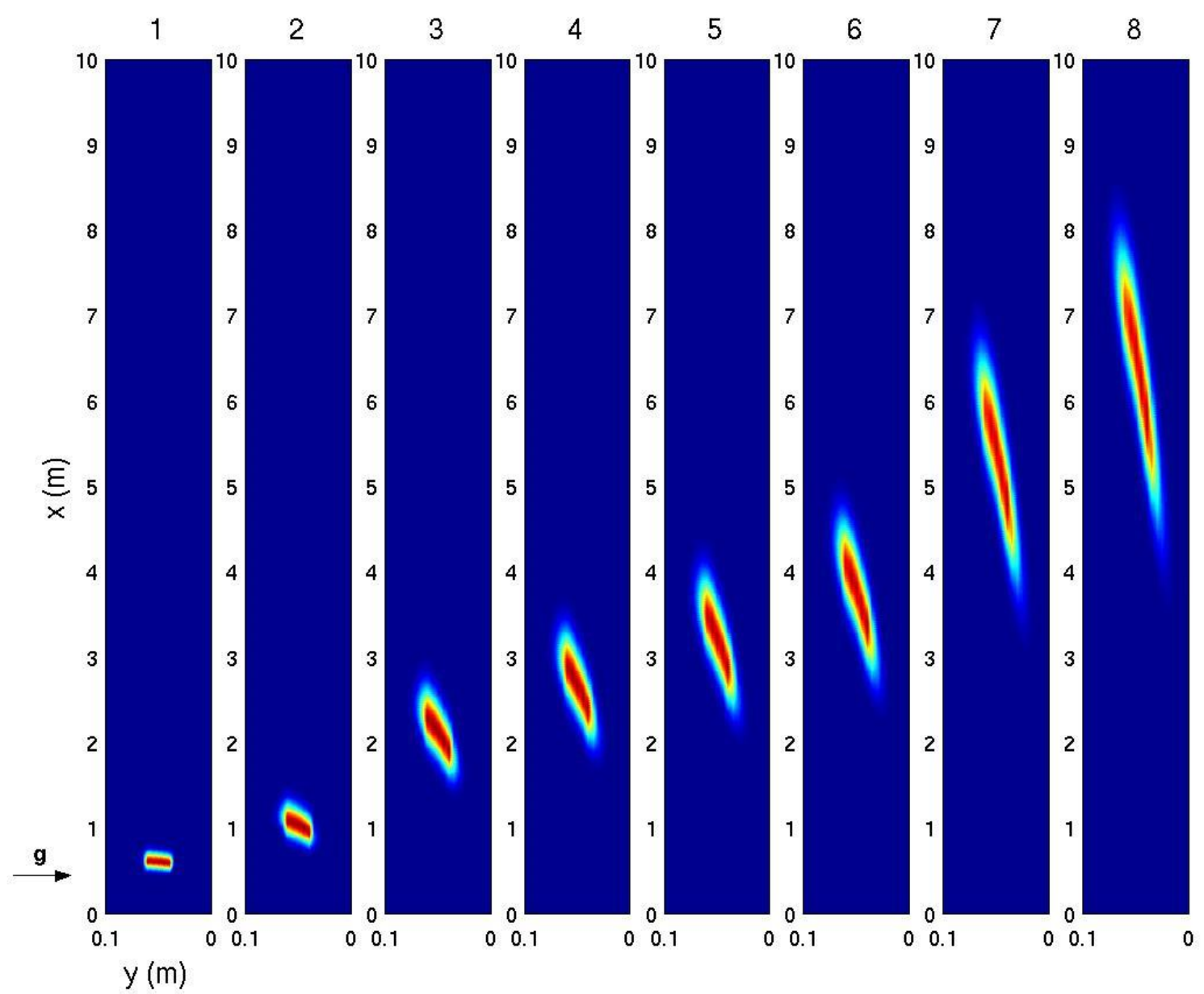

Figure 8 Channel flow $(d=0.5, L=10 \mathrm{~m})$. Snapshots in time ( $t=5 \mathrm{~s})$ of concentration contours of a spot

( $44 \mathrm{~cm}$ ) of particle size $a=50 \mathrm{~m}$. Inlet flow velocity is $\mathrm{U}_{0}=0.17 \mathrm{~m} / \mathrm{s}$, from bottom to top. Bingham viscosity is ${ }_{p}=0.268 \mathrm{Pas},{ }_{0}=2.67 \mathrm{~Pa},{ }_{m}=0.68, \quad=2,790 \mathrm{~kg} / \mathrm{m}^{3}$. The authors show the sheared layer $\left(\begin{array}{lll}y & 0.1 & \mathrm{~m}\end{array}\right)$ 
This experiment made evident the shear properties of the boundary layer created by the effect of the bottom wall and the main stream. The local shear turns on the Phillips fluxes and the diffusion of the particle spot creates an inclined high concentration line which evolves downstream but approaches the bottom wall extremely slowly, because of the particle size.

The Phillips fluxes must be strong enough to overcome the local stress and gravitational forces, otherwise sedimentation takes place and the spot will progressively approach the bottom wall before reaching the channel exit.

Theory predicts a competition between all involved particle forces, especially the migration from high to low shear regions as a product of Phillips fluxes (varying viscosity and collision frequency). In Figure 8 the authors observed an almost equilibrium condition between these forces, as the spot never reaches the bottom wall because the Phillips fluxes are strong enough to slow down sedimentation.

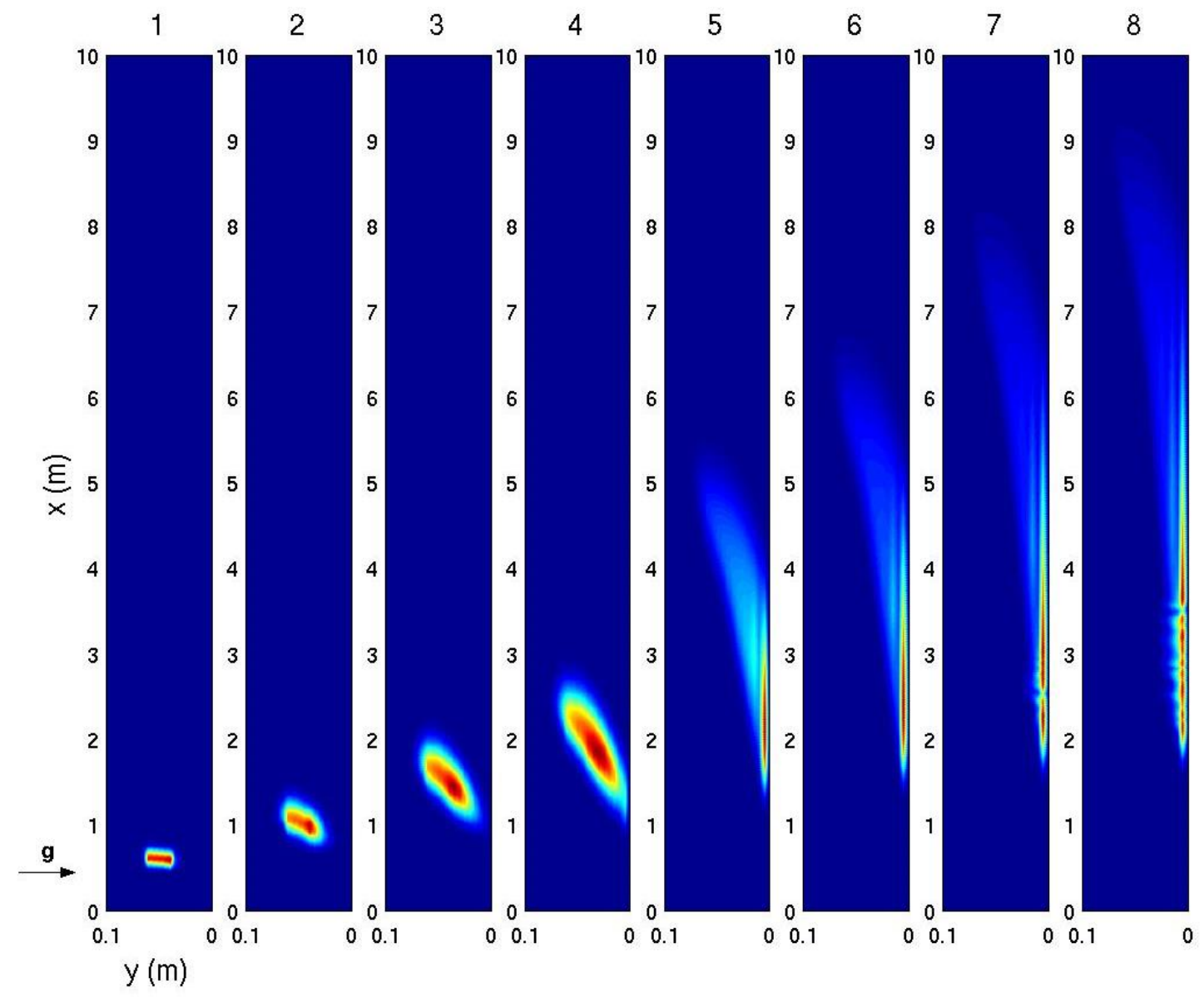

Figure 9 Channel flow $(d=0.5, L=10 \mathrm{~m})$. Snapshots in time $(t=5 \mathrm{~s})$ of concentration contours of a spot

(4 $4 \mathrm{~cm}$ ) of particle size $a=200 \mathrm{~m}$. Inlet flow velocity is $\mathrm{U}_{0}=0.17 \mathrm{~m} / \mathrm{s}$, from bottom to top. Bingham viscosity is ${ }_{p}=0.268$ Pas, ${ }_{0}=2.67 \mathrm{~Pa},{ }_{m}=0.68, \quad=2,790 \mathrm{~kg} / \mathrm{m}^{3}$. The authors show the sheared layer $\left(\begin{array}{lll}y & 0.1 \mathrm{~m}\end{array}\right)$

One way to get stronger Phillips fluxes is to increase the inlet velocity and therefore the shear rate $[$ rate across the entire channel (note that $\sqsubset$ is maximum at $y=0$ ). However, if the authors want to keep a similar hydrodynamic state, they should increase the particle size, whereas when the authors consider coarser particles (e.g. size $\mathrm{a}=200 \mathrm{~m}$ ), they do increase both gravitational and Phillips fluxes. This leads to a combined advection and sedimentation dynamic, as the localised spot progressively approaches the bottom wall of the channel (Figure 9) accompanied by a strong spot spreading across the wall, creating a 
bed-like structure. This is very important in regard to slurry flow solids transport technology, as the model is able to predict the formation of a bottom bed as shown in the last snapshot sequence of Figure 9 .

\section{Conclusions}

The authors solved the laminar hydrodynamics and particle concentration dynamics in highly concentrated non-Newtonian slurry laminar channel and pipe flows. The authors numerically solved a $2 \mathrm{D}$ model of the Navier Stokes and mass conservation equations coupled with a novel scalar concentration model to describe the behaviour of coarse mono-modal particles in laminar non-Newtonian flows. The scalar concentration equation predicts the volume fraction of solid particles and allows computation of the apparent viscosity of the fluid as a function of both local solid concentration and local shear, which is finally considered as the input in the stress tensor of the Navier Stokes equations.

Our results are in good agreement with similar models and experiments found in the literature, as the results of the numerical simulation show that particle migration effectively occurs from high to low shear regions, provided that the new fluxes in the scalar concentration equation are stronger than the critical yield stress of the slurry or gravitational effects.

The model predicts the initial formation of a kind of bottom bed for a determined flow velocity, particle size and average concentration. Future work is concerned with the sliding motion of such bed-like structures and the change in effective channel depth.

\section{Acknow l edgements}

First author acknowledges financial support from Fondecyt Grant Nº 1085020.

\section{References}

Beverly, C.R. and Tanner, R.I. (1992) Numerical analysis of three dimensional Bingham plastic flow, in Journal of Non-Newtonian Fluid Mechanics, Vol. 42, pp. 85-115.

Hernández, R.H. (1995) Influence of the heating rate on supercritical Rayleigh-Bénard convection, International Journal of Heat Mass Transfer, Vol. 38 (16), pp. 3035-3051.

Krieger, I.M. (1972) Rheology of monodisperse lattices, Advances in Colloid and Interface Science, Vol. 3, pp. 111-136.

Mills, P. and Snabre, P. (1995) Rheology and structure of concentrated suspensions of hard spheres, Shear induced particle migration, in Journal de Physique, II France, Vol. 5, pp. 1597-1608.

Patankar, S.V. (1980) Numerical Heat Transfer and Fluid Flow, Hemisphere Publishing Corporation, Washington DC.

Phillips, R.J., Armstrong, R.C., Brown, R.A., Graham, A.L. and Abbott, R. (1992) A constitutive equation for concentrated suspensions that accounts for shear-induced particle migration, in Physics of Fluids A, Vol. 4 (1), pp. 30-40.

Schaan, J., Summer, R.J., Gillies, R.G. and Shook, C.A. (2000) The effect of particle shape on pipeline friction for Newtonian Slurries of fine particles, Canadian Journal of Chemical Engineering, Vol. 78, pp. 717-725.

Spelay, R.B. (2007) Solids transport in laminar, open channel flow of non-Newtonian slurries, A Thesis Submitted to the College of Graduate Studies and Research in Partial Fulfilment of the Requirements for the Degree of Doctor of Philosophy in the Department of Chemical Engineering University of Saskatchewan Saskatoon, Saskatchewan, Canada, $446 \mathrm{p}$.

Van Doormaal, J.P. and Raithby, G.D. (1984) Enhancements of the simple method for predicting incompressible fluid flows, Numerical Heat Transfer, Vol. 7, pp. 147-163. 